\title{
Fuzzy logic in construction management
}

\author{
Fjoder Klashanov ${ }^{1, *}$ \\ ${ }^{1}$ Moscow State University of Civil Engineering, Yaroslavskoye shosse, 26, Moscow, 129337, Russia
}

\begin{abstract}
The article is devoted to the development of a model of management of technological processes of construction on the basis of fuzzy logic. Construction is a complex open dynamic systems with elements of ambiguity and uncertainty. External factors are not always possible to clearly determine the relationships between the factors are constantly changing in a random way. The dynamics of the laws describing the external and internal parameters of the system of control over the construction process and their relationship with the growth of scientific and technical progress only increases. This is due to the emergence of new information flows, which depends both on internal and external multiple factors with elements of ambiguity and chance, but we need to make, to store, quickly process and transmit information for the elaboration and adoption of decisions.
\end{abstract}

\section{Introduction}

Construction is one of the most important sectors of the economy. States of construction largely determines the level of development of society and participate in it: investor, customer, designer, contractor, specialized building organizations, enterprisesmanufacturers of appropriate building materials, machinery and technological equipment. Thus, the construction process is influenced by many factors, which are fuzzy and/or not fully defined.

Information about construction at all stages is associated with unclear defined as external and internal factors. To find the optimal process of construction management, it is necessary to rely on modern computer technologies that make it possible to increase the level of automation of construction management. The use of computer technology requires the necessary formalization of the factors that determine the process of construction management. Development of management solutions in the construction industry using modern computer technology and taking into account incomplete or fuzzy information should be based on the methods of artificial intelligence. The most appropriate method in this case is a method that uses fuzzy sets and fuzzy logic.

\section{Materials and methods}

\footnotetext{
* Corresponding author: klashanov@mgsu.ru
} 
Almost any scope can have one solution, but several. Before a technologist is always the problem of choosing the most rational way to achieve a goal, usually experience tells him what decision is most beneficial under the given production conditions. Attempt to use the first order logic for knowledge representation in problem areas such as the technology of instrumentation, may fail for two main reasons, described below [1].

First, the formation of a complete set of antecedents or consequental necessary for drawing up rules that have no exceptions too labor-intensive, and the application of these rules is too complex.

Second, even if you know all of the rules, may remain uncertainty about the solution to a specific technological problem, because the production situation may significantly change, for example, due to unforeseen failure of necessary equipment.

Although the tasks and heterogeneous, but they share one common feature - they are all clearly presented and require for their solution not the traditional classical methods based on two-valued logic that is implemented in cybernetic circuits, and the use of fuzzy sets and multivalued logic. This approach would allow ill-defined problem translated into the language of computer technology and a variety of management options to the decision maker to choose the best.

A solution that would accommodate a number of contradictory requirements such as input of objects in operation in target dates with appropriate quality, required efficiency, taking into account scientific and technical progress and productivity growth. Here it is expedient to apply the methods of fuzzy sets and fuzzy logic. The sequence of actions is as follows. Initially, the problem is described by the words of the spoken language, where the sentences are predicates of the first order. Then identify the parameters that characterize the problem and define linguistic variables [2].

Fundamentally linguistic variable differs from numerical variable because its values are not numbers but words or, in extreme cases, the proposals outlined in natural language. Linguistic variable enables us to describe a complex situation that cannot be described in conventional quantitative terms, and analyzed a highly efficient methodology using the theory of multivalued logics and fuzzy sets.

Thus, in verbal description, is developed by a logical-linguistic model in a complex nondeterministic domains with fuzzy logic and fuzzy definitions followed by the construction of a task tree that allows you to formalize and develop a mathematical model for further automation on the basis of a computer search of the optimal variant [3].

Fuzzy set relative to the linguistic variable plays a role similar to that played by the words in a sentence in a natural language. A linguistic variable is defined as a tuple $<\mathrm{X}, \mathrm{T}(\mathrm{X}), \mathrm{U}, \mathrm{G}, \mathrm{M}>$, where $\mathrm{X}$ is the variable name; $\mathrm{T}(\mathrm{X})$ be the set of its values (the base term-set), consists of the names of fuzzy variables, domain of definition of each of which is a multiple of $\mathrm{U} ; \mathrm{G}$ - syntactic procedure (grammar), allowing the use of elements of termset T; $\mathrm{M}$ - semantic procedure, which allows to assign to each new value of the linguistic variable the fuzzy semantics through the formation of a new fuzzy set. The semantic procedure allows you to set the function of truth $\mu(\mathrm{u})$, where, $0 \leq \mu(\mathrm{u}) \leq 1$.

Fuzzy logic provides a highly effective solution to the problem, given fuzzy or incomplete information. It allows you to design multivariable regulators with Supervisory functions not on the basis of mathematical methods, and on the basis of experimental data and experience of the operators.

Discusses the main directions of application of some methods of fuzzy sets and fuzzy logic in designing control systems in the construction industry because the construction industry is associated with clearly defined both external and internal factors. Examples of specific management objects, as well as describes the General algorithm for the main stages of the process, fuzzy control [4]. 
The construction industry is the most significant and important for the successful development of which it is necessary to apply the achievements of scientific and technical progress. society. Therefore, in the modern world to the construction industry is presented with more and more requirements. Construction projects must meet all of the requirements, meets the comfort, security, sustainability and efficiency.

If the system is complex and/or background information is unclear or not well defined, the whole process of management can be described in natural language terms instead of strict mathematical formulation and two-valued logic, use the methods of fuzzy sets and multivalued logic. Therefore, before the decision maker, the problem of choosing the optimal ways of achieving the goal. To enforce these vague, incomplete and somewhat contradictory requirements control system construction should take into account the modern achievements of scientific and technical progress. Making the right decisions in the management of construction operations for incomplete or fuzzy input information is one of the most difficult but important tasks in the organization of construction [5]. Therefore, it is advantageous when constructing fuzzy control systems to use not only expert knowledge, but also the basic concepts of the theory of automatic control.

To give General recommendations concerning the choice of a basis is not possible - it all depends on the specifics of the managed object. It should be noted that in many publications on the basis of limited experimental data it is concluded that fuzzy algorithms provide higher efficiency compared to classic. This is not always true [6]. In each case it is necessary to compare the required quality of control with available resources. The emergence of the theory of fuzzy sets and control algorithms based on it are caused, primarily, by the appearance of a task for which traditional mathematical apparatus simply does not exist.

The efficiency of the use of soft computing is very high. This can be seen in numerous publications on this topic. However, this efficiency is markedly increased if, in addition, soft computing is to apply traditional methods of theory of automatic control, tested for decades and have a fairly rigid mathematical basis.

The primary variable characterizing the quality of the process is the content of heavy metal ions in the effluent at the outlet of the cleaning apparatus. Existing tools measure the concentrations of heavy metal ions is complex and expensive to operate, do not provide the necessary for process control the accuracy and frequency of measurement. In addition, small changes of the input variables of the process can dramatically change the parameters of output variables. Therefore it is necessary to define a measure of the quality of the wastewater or the contents of heavy metal ions in them.

A situation in which it is desirable to translate the object, i.e. the target situation is determined based on the analysis of the degrees of preference of operating decisions. The degree of preference of the latter depend on specific situations and are defined as a production system (rule set) "situation - preference decisions" [7].

Information flow serves the basic functions of enterprise management: forecasting, planning, organization, management, coordination, control, decision making etc. it gives information about the flow of material, but not in the form of primary documents, and in the form obtained on the basis of the consolidated (aggregated) indicators of economic activity of the enterprise. The provision of such data in the information system is the basic function of accounting (financial and managerial) accounting, and economic analysis. On the basis of the aforementioned information out of the company's development strategy, which together with other data such as marketing data analysis and forecast of the major markets for products of the enterprises are the basis for planning activities of the enterprise.

Decisions on the construction and modernization refer to the strategic management level and should ensure the technological and competitive advantages of the enterprise. The implementation of these solutions is based on approved estimates and budgets for 
construction projects. After the completion of the issued acts of acceptance, and the fixed assets are commissioned. Important information flows support the process of personnel management of the enterprise. In them there is a flow in the system of accounting information on the amount of time actually worked, is payroll, pension and other social security contributions. Based on this information, you can build the logical structure of the information system of the enterprise.

When developing management decisions, it is necessary to take into account factors such as unreliability and incompleteness of information, and Vice versa, possible redundancy and heterogeneity of information. Some of the information is questionable and sometimes false, and therefore requires strict control and full verification.

To solve this problem and is called fuzzy logic. The term "linguistic variable" can be linked to any physical quantity for which you want to have more values than just Yes and no. In this case, is determined by the required number of terms and each of them corresponds to some value described by physical quantities. For this value the degree of belonging to the physical quantity term is equal to unity and all other values will depend on the chosen membership functions.

For the description of linguistic variables, terms and fuzzy rules uses a special language: Fuzzy Control Language (FCL) described in the standard IEC 1131. Most developed of all the development of artificial intelligence expert system gained a strong recognition as a systems support decision-making.

One of the main methods of knowledge representation in expert systems is production rules that allow to approach the style of human thinking. Any rule consists of premises and the conclusion. There may be multiple parcels in the rule, in this case they are combined by logical connections and - or .

The main drawback of production systems is that they require the availability of complete information about the system. Fuzzy systems are also based on production rules, but as a parcel and the conclusion of a rule uses linguistic variables, thus avoiding limitations inherent in the classical production rules.

Target setting management process associated with the output variable fuzzy control system, but the result of fuzzy inference is fuzzy, and the physical actuator is not able to perceive such a team. Requires special mathematical methods to transform fuzzy values to very specific. The whole process of fuzzy control can be divided into several steps: fuzzification, development of fuzzy rules and defuzzification [8].

\section{Results}

Fuzzy system copes with this task very quickly, despite the fact that instead of complex differential equations of motion of the work piece, the whole process of motion is described by natural language terms: "more", "medium", "slightly", etc. — like robot controlled by the operator.

Fuzzification (transition to fuzziness). The exact values of the input variables are converted into values of linguistic variables through the application of certain provisions of the theory of fuzzy sets, namely through certain membership functions. Consider this stage in more detail. First of all, we introduce the concept of "linguistic variable" and "membership function".

In fuzzy logic the values of any magnitude are represented not by numbers, but by words of natural language are called terms. So, the value of the linguistic variable distance are the baths far, close, etc. To implement a linguistic variable necessary to determine the precise physical meaning of its terms. Suppose, for example, a variable distance can be set to any value from 0 to 50 meters. According to the theory of fuzzy sets, each distance value from a range of 50 meters can be assigned to a number from zero to one, which determines 
the degree of belonging of the physical distance values (for example, $10 \mathrm{~m}$ ) to a particular term of a linguistic variable distance. In our case the distance of 40 meters you can set the degree of belonging to term far 0.85 , and the Terme is close to 0.15 . Specific definition of degree of membership is possible only when you are working with experts (in our case, technologists and designers). Currently have the impression that for most applications it is sufficient 3-7 of terms for each variable.

As already mentioned, the exact membership of each value to one of the terms of a linguistic variable is defined by a membership function. Its appearance can be absolutely arbitrary. Now we have formed the concept of the so-called standard membership functions, including functions such as Z-function, S-function, triangular function and trapezoid function. Standard features well suited for solving most common tasks.

To solve more complex tasks may require the creation of a special membership functions, for example, polygon or single. Development of fuzzy rules. Most of fuzzy systems use production rules to describe dependencies between linguistic variables. A typical rule consists of the antecedent (if ...) and consequent (then part ...). The antecedent may contain more than one parcel. In this case, they are combined by logical connections and/or.

In the first step of logical inference is necessary to determine the degree of belonging of all antecedent rules. To do this, in fuzzy logic there are two operators: $\operatorname{MIN}(\ldots)$ MAX(...). The first calculates the minimum value of degree of membership, the second the maximum. When to apply a particular operator, depends on a bunch of connected parcels in rule. If you use the bundle and apply the operator $\mathrm{MIN}(\ldots)$. If parcels are combined by the conjunction or, it is necessary to apply the operator $\operatorname{MAX}(\ldots)$.

Defuzzification (addressing vagueness). In this step, the transition from the fuzzy values of certain physical parameters which can serve as a command to the Executive device. The result of fuzzy inference, of course, will be fuzzy. In the example with the robot command for the electric motor will be presented term average (power), but the actuator is really nothing, therefore, to eliminate fuzziness may apply special mathematical methods (e.g., method of center of maximum), allowing the output to obtain the exact values that are passed directly to the Executive device of the manipulator.

The formation of the structure of the information system of the modern industrial enterprise should start with a qualitative analysis of the information field of the enterprise, which can be divided into internal and external. Internal information field comprises the following information: primary documents; internal document management (paper and electronic), including orders and instructions of the Director and managers of all units; financial reporting and other mandatory reporting for the current and prior periods; analysis of financial and economic activities; other data.

\section{Discussions}

The quality of the internal information field of the enterprise depends on the definition of the organizational management structure, the rational allocation of functional responsibilities, reliability of accounting, sufficient efficiency of the scheme document. Internal information field is formed at the expense of own sources of information that can be checked for completeness and accuracy.

Number of varieties of external information and its sources very much, the main are the following: regulations of the Federal, regional and local level; industry-standard reference documents; data on the state of industry, key markets and raw materials; data on the state of the world economy; advertising and information partners and competitors; information from customers; insights of consultants and experts, the results of audits. 
It is necessary to distinguish the following main problems encountered when creating the external information field.

1. The inaccuracy of information. Some of the information (especially in the media and on the Internet) is questionable, and in some cases false.

2. The incompleteness of information. Source of information intentionally or unintentionally may not provide all the information, and some part of it.

3. The contradictory information. Information of different sources may be contradictory, in which it is difficult to identify the true data.

4. The redundancy of information. To provide the necessary information needed to expend considerable effort.

5. The heterogeneity of information. Information from different sources in different format. For the harmonization of the information for further storage and processing by a single technology it should be converted.

\section{Conclusion}

Fuzzy logic provides a highly effective solution to the problem, given fuzzy or incomplete information. It allows you to design multivariable regulators with Supervisory functions are not based on mathematical methods, and on the basis of experimental data and experience of other researchers. The verbal description of the construction management process, including the ambiguity and randomness of both external and internal factors is the block description of the control system construction. Logical-linguistic model allows to develop and to find the optimum option of the construction management system

\section{References}

1. D.A. Pospelov, Logic-linguistic models in control systems (Energoizdat, Moscow, 1981)

2. F.K. Klashanov, Vestnik MGSU 1(1), 331-338 (2011)

3. F.K. Klashanov, To theoretical base of the building to models of management in construction (2014) conferences.dce.ufl.edu/ICCCBE2014

4. A.A. Volkov, S.N. Petrova, Information systems and technologies in construction (MGSU, Moscow, 2015)

5. K.V. Postnov, Diversification as a tool for developing the project organization 4-5, 129-136 (2010)

6. N. Ivanov, Vestnik MGSU 5, 326 (2011)

7. F. Aggogeri, A. Borboni, R. Faglia, A. Merlo, S. De Cristofaro, Applied Mechanics and Materials 336-338, 1170-1173 (2013) doi : 10.4028/www.scientific.net/AMM.336338.1170

8. A. Borboni, F. Aggogeri, R. Faglia, Journal of Advanced Robotic Systems 10, 149 (2013) doi : 10.5772/55539

9. B.A. Kulik, A.A. Zuenko, A.Ya. Fridman, Algebraic approach to intelligent data processing and knowledge (Publishing house of Polytechnical Institute. University press, $\mathrm{SPb}, 2010$ )

10. F.K. Klashanov, Scientific review 14, 370-374 (2015) 